\title{
The Effect of Group Reminiscence Therapy on Insomnia in Older Adults: A Randomized Controlled Clinical Trial
}

\section{Fatemeh Sahragard ${ }^{1}$, Mahboubeh Rezaei ${ }^{* 2}$, Fatemeh Sadat Izadi-Avanji ${ }^{1}$, Fatemeh Atoof ${ }^{3}$, Maryam Ahmadishad ${ }^{1}$}

1- School of Nursing and Midwifery, Kashan University of Medical Sciences, Kashan, Iran.

2- Autoimmune Disease Research Center, Kashan University of Medical Sciences, Kashan, Iran.

3- School of Health, Kashan University of Medical Sciences, Kashan, Iran.

*Correspondence: Autoimmune Disease Research Center, Kashan University of Medical

Sciences, Kashan, Iran. Tel: +983155540021. Email: m4.rezaei@gmail.com.

Received July 15, 2019; Accepted December 17, 2019

\section{Abstract}

Background: Insomnia is one of the common sleep disorders in the elderly. Lack of adequate sleep has many consequences, such as fatigue, frustration and increased rate of mortality among them. The study was carried out to determine the effect of group reminiscence therapy on insomnia in older adults.

Methods: This randomized controlled clinical trial was done on 72 older adults who referred to Sadoughi health care center in Kashan, Iran between August and November 2018.The subjects were recruited through convenience sampling and randomly allocated into two control and intervention groups. The reminiscence therapy was held twice a week, in a 4-week period for intervention group and the duration of each session varied between 1.5-2 hours. There was no intervention in control group. Data were collected using Mini-Mental State Examination (MMSE), and Insomnia Severity Index (ISI). Data was gathered three times; before intervention, immediately and one month after the intervention. Data were analyzed using Chi-Square, and Man-Whitney tests and repeated measures analysis in SPSS (v. 16.0, SPSS Inc., Chicago, IL, USA).

Results: The mean score of insomnia decreased in intervention group after group reminiscence therapy ( $\mathrm{p}<0.001$ ); from $16 \pm 1.74$ (before the intervention) to $11.27 \pm 2.09$ (immediately after the intervention) and $11.38 \pm 1.93$ (one month after the intervention), while the insomnia score had not noticeable change in control group. The intervention group showed significantly less insomnia scores over the time compared to control group $(\mathrm{p}<0.05)$.

Conclusions: Reminiscence therapy decreases the severity of insomnia in older adults. 
Therefore, it can be used as an easy, applicable and low-cost technique in health care facilities and is expected to be welcomed by older adults, their families and health care providers.

Keywords: Sleep, Insomnia, Reminiscence Therapy, Geriatrics, Randomized controlled trial 10.29252/jgbfnm.17.1.94

\section{Introduction}

Sleep is one of the most important human circadian rhythms and biological patterns (1). It is one of the basic human needs that is essential for maintenance of energy and appearance as well as physical and psychological wellbeing (2). The desirable sleep preserves physical health, reduces the boredom and anxiety, improves the ability to adapt and focus on activities of daily living. On the other side, sleep deprivation leads to fatigue, headache, disruption of concentration, drowsiness that may increase the rate of mortality $(3,4)$. In the elderly, the insomnia prevalence is higher than in the younger population. The prevalence of insomnia symptoms in the elderly ranged from $30 \%$ to $48 \%$ (5). A study reported the prevalence of more than $\% 57$ and the incidence of $50 \%$ for insomnia in the elderly (6). Insomnia is broadly defined as dissatisfaction with sleep either qualitatively or quantitatively. This is usually associated with one or more of the following: a) difficulty initiating sleep, b) difficulty maintaining sleep characterized by frequent awakenings or problems returning to sleep after awakenings, and c) early-morning awakening with inability to return to sleep (7). Stressful life or medical conditions, physical disability, and fair to poor perceived health, medications such as beta blockers, glucocorticoids, non-steroidal anti-inflammatory drugs may be one of the factors contributing to insomnia $(8,9)$.

The older adults often relate these disorders to the normal process of aging and rarely seek treatments $(10,11)$.

Older people often take sleeping pills for sleep disorders so that $39 \%$ of sleep medications are used by people aged over 65. These kinds of medications improve the sleep quality temporarily and most of them reduce quick eye movements that are necessary for brain performance and alleviation of the stress (12). Another way for improving the sleep quality is using nonpharmacologic interventions that don't have side effects such as drug resistance and drug dependence (13). One of the non-pharmacologic interventions is group reminiscence therapy. It is often used for older adults and is based on reminding the past events, feelings and thoughts in 
order to bring them pleasure and excitement and to improve the quality of life and compatibility with their current situation $(14,15)$.

In reminiscence therapy, an older adult reviews his or her entire life with the focus on Erikson's eight stages (16) and expands his perception towards the meaning of life by restoration of the events and placing them in a framework (17). This type of psychotherapy facilitates the coping strategies by improving cognitive organization (18). Reminiscence therapy may result in life satisfaction, improving the quality of life, reducing the depression, stress and anxiety as well as helping the socialization in older adults (19). There are different types of reminiscence therapy such as integrative, instrumental, narrative, obsessive and escapist reminiscence. The obsessive and escapist reminiscences not only are not therapeutic but also have a destructive effect. Instead, the integrative, instrumental and narrative reminiscence can be used for different therapeutic objectives (20).

Among different types of reminiscence therapy, the narrative one is descriptive and its purpose is to communicate with others. It raises the positive feelings in social interactions and leads to life satisfaction (21). During the reminiscence, the participant devotes more time on remembering his/ her past consciously. Furthermore, reminiscence may have a positive impact on the person's temper. The older adults devote a few hours of daytime to sleeping because they try to think and remember the past events during the entire day (22). The results of various studies show that reminiscence therapy can alleviate the depression (23-25), anxiety (23) and loneliness (26, 27) and enhance the self-confidence $(24,28)$ and vitality $(29)$. Another study reported that group reminiscence therapy had significant effect on improving the mental health and quality of sleep in older adults [30]. However, there are some studies reporting that this intervention has no effect on the sleep disorders. Results of one study showed multiple awakenings during sleep are happened after six session group reminiscence therapy in older adults (31). Also, another study reported that before and after the reminiscence therapy no change was observed in sleeping of older adults with Alzheimer disease (32).

In one study, both laughter therapy and group reminiscence therapy had significant impact on sleep but laughter therapy was more effective on sleep quality in the elderly (33).

Given the results of various studies about the effect of reminiscence therapy on different dimensions of mental health, a limited number of researches have reports about the effects of reminiscence therapy on sleep and specially insomnia in the older adults along with its side 
effects and consequences as well as its cost-effectiveness with respect to the cultural differences. Here we aimed to evaluate the effect of group reminiscence therapy on insomnia in older adults.

\section{Methods}

This randomized controlled clinical trial was done on 72 older adults in Kashan, Iran in 2018. The eligible older adults, who referred to Sadoughi health care center as a referral center in south of Kashan, were invited to participate in the study from August to November.

According to the finding of similar study (34) and with an alpha error of 0.05 and a statistical power of $90 \%$, a sample size was calculated at least 29 in each group. Considering a possible sample attrition rate of $20 \%, 72$ older adults were recruited to the study. The participants were selected by convenience sampling considering inclusion criteria $(n=80)$. Then 72 eligible participants were randomly assigned to control $(n=36)$ and intervention $(n=36)$ groups according to predesigned blocks with a block size of 4 . In this type of randomization, there are six possible permutations with balanced group allocation. At first, the list of blocks was specified, and numbers 1 to 6 were assigned to these blocks. Then numbers 1 to 6 were randomly selected from a table of random numbers, and a sequence of A and B were formed. Participants were assigned to intervention (A) and control (B) groups according to the randomly selected block. Four participants did not have inclusion criteria and therefore they were excluded from the study. (Fig. 1)

The inclusion criteria were as follows; age 60 and over, hearing and speech ability, ability to write and read, having good physical ability to take part in sessions, 22 or greater score in Mini Mental Examination (MMSE) (35), 15 or greater score in Insomnia Severity Index (ISI) (36), taking no sleeping pills, not having depression disorder and chronic cardiac and respiratory disease. 


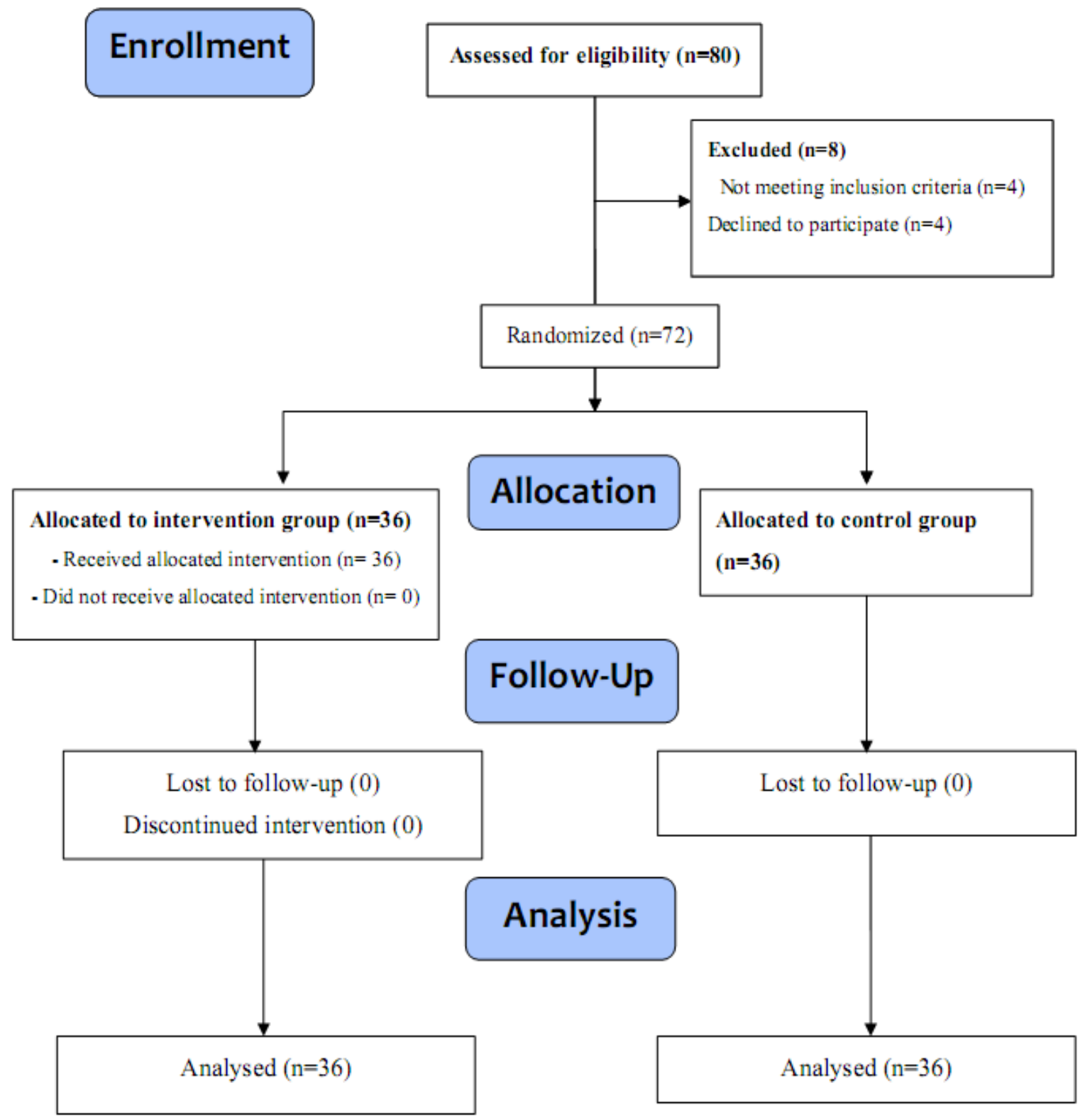

Figure 1. Consort flow diagram of participant enrollment, allocation and follow up

The study was approved by Ethics Committee of Vice Chancellor for Research, Kashan University of Medical Sciences (ethical code: IR.KAUMS.NUHEPM.REC.1397.022). All participants received the information and objectives of the study and were assured that their information would remain confidential. All participants signed the informed consent form. Also, 
this trial was assigned the Iranian Randomized Controlled Trial Registry (ID: IRCT20180722040553N1).

Data was collected using questionnaires; including demographic characteristics (age, gender, marital status, educational level, occupation, financial status, real state ownership, living situation, number of children and history of chronic disease), Mini-Mental State Examination (MMSE), and Insomnia Severity Index (ISI).

The MMSE is a 30-point questionnaire that is developed and validated by Folstein et al. (1975) (35) to assess the patients' cognitive functions. The MMSE has 10 points for orientation, 6 points for memory, 5 points for attention and calculation, 8 points for language, and 1 point for visouspatial abilities. The cut-off score of 22 point was established by ROC Curve analysis to detect cognitive impairment (sensitivity $=90 \%$, specificity $=93.5 \%$ ). The validity and reliability of the MMSE were evaluated by Seyedian et al. (2008) (37) in Iran and its internal consistency was represented by the Cronbach's alpha level of 0.81 .

The ISI developed by Morin et al. (1999) (7), is a 7-item self-report instrument that measures the severity of the patients' insomnia. This questionnaire is designed to evaluate severity of the problems in sleep onset and sleep maintenance (nighttime and early morning awakening problems), sleep dissatisfaction, interference of insomnia with daytime functioning, noticeability of problems associated with sleep disorders, and distress caused by the sleep disorders. A 5-point Likert-type scale is used to rate each item from 0 (no problem) to 4 (severe problem). The total scores of the questionnaire range from 0 to 28; so the higher scores indicate the more severe sleep problems. The total score is interpreted as follows: absence of insomnia (0-7), sub-clinical insomnia (8-14), moderate clinical insomnia (15-21), and severe clinical insomnia (22-28) (7, 38). The validity and reliability of ISI were evaluated by Yazdi et al. (2012) in Iran and the Cronbach's alpha was presented higher than 0.8 (39).

In this study, the reminiscence therapy sessions were held in the morning in a well-lighted and ventilated room at the Sadoughi health care center in Kashan. For setting the intervention group, researchers divided the participants into same six groups. Initially to start therapy sessions, researchers arranged material pertaining to the reminiscence therapy based on Stinson \& Kirk (2006) approach considering Iranian context and culture. Then the sessions were held twice a week in a 4-week period for all six groups (40). The duration of the sessions varied between 1.52 hours with respect to the topic of the session (Table1). According to Stinson \& Kirk protocol, 
the participants merely expressed their memories. They believe that since the topic and structure are predetermined in the structured reminiscence therapy, the participant follows a specified framework for recalling and reviewing his/her memories and is prevented from negative reminiscing. Along with reminiscing about the past, the participant is guided to a combination of positive reminisce such as the narrative, integrative and instrumental reminiscences (40).

The day before each session the participants were called and reminded about the time and topic of that session. At the beginning of the session, the time duration was determined for each participant so that all of them had enough time for expressing their memories. The group members were committed to listening and also advised to avoid any judgment of each other. At the end of every session, the leader (first author) appreciated the participant attendance and asked them to be prepared for the next session. Before the beginning study, the first author became familiar with the structured reminiscence therapy protocol and also consulted with a researcher who had previously worked in this field. She was as a leader and facilitator and prevent that participants express their negative memories and contents that are irrelevant to the topics.

Table 1: The topics of structured reminiscence therapy sessions

\begin{tabular}{|c|l|}
\hline Session & \multicolumn{1}{|c|}{ The topic of the session } \\
\hline $\mathbf{1}$ & $\begin{array}{l}\text { Getting information about the aims and methods, getting acquaintance with each } \\
\text { other, completing the questionnaires }\end{array}$ \\
\hline $\mathbf{2}$ & Expression of childhood memories \\
\hline $\mathbf{3}$ & Expression of youth and education memories \\
\hline $\mathbf{4}$ & Expression of memories about marriage and birth of the children \\
\hline $\mathbf{5}$ & Expression of memories about work experiences and successes \\
\hline $\mathbf{6}$ & Expression of memories about celebrations and travels in different periods of life \\
\hline $\mathbf{7}$ & Expression of memories about important and special events of life \\
\hline $\mathbf{8}$ & Summing up and filling the questionnaires \\
\hline
\end{tabular}

Firstly, the cognitive function of the eligible older adults was considered using MMSE (35). The elderly who obtained a MMSE score of more than 22 were selected. In the next step, the selected ones who obtained an ISI score of more than 15 (moderate clinical insomnia) were recruited to the study. At the beginning of the first session the subjects completed the "participants' characteristics questionnaire" and ISI questionnaire (38). The ISI was also completed 
immediately (one night after the last session) and one month after the intervention as follows up. For control group, there was not any intervention and same as measurement in intervention group, the questionnaires were completed at baseline and immediately and one month after that. All the questionnaires in the intervention and control groups in three times were self-reported and were completed by the participants in the Sadoughi health care center.

SPSS for Windows (v. 16.0, SPSS Inc., Chicago, IL, USA) was used for data analysis. Data were presented using descriptive statistics measures (such as mean, standard deviation, median) for quantitative variables and frequency distribution for qualitative variables. To ensure that the data were normally distributed, Shapiro-Wilk test was carried out.

In order to assess demographic characteristics in two groups, Chi-Square test was used for qualitative variables and Man-Whitney test was used for the age as a quantitative variable. The Independent samples t-test was also applied to compare the mean scores of the insomnia between the two groups at the beginning, immediately after the intervention and one month after the end of the study. In order to evaluate the effect of reminiscence intervention on insomnia over the time, repeated measures analysis was used. Moreover, the Mauchly's W test was used to examine sphericity. $\mathrm{p}<0.05$ was considered as statistically significant.

\section{Results}

The findings showed that the median age of patients were 62 (60-67) years. Majority of the patients were female $(66.7 \%)$, married $(84.7 \%)$ and with primary education $(80.6 \%) .87 .5 \%$ of the participants had not any history of chronic disease. There were no significant differences regarding participants' characteristics between intervention and control groups at baseline $(\mathrm{p}>0.05)$. Only there was a significant difference between financial status of the participants in both groups ( $\mathrm{p}=0.01)$. More details on participants' characteristics were presented in Table 2.

\section{Table 2. The characteristics of participants in intervention and control groups}




\begin{tabular}{|c|c|c|c|c|}
\hline \multirow{2}{*}{\multicolumn{2}{|c|}{ Variable }} & \multicolumn{2}{|c|}{ Group } & \multirow{4}{*}{$\begin{array}{c}\text { P-value } \\
1\end{array}$} \\
\hline & & \multirow{3}{*}{$\begin{array}{c}\begin{array}{c}\text { Intervention } \\
\mathbf{N}(\%)\end{array} \\
24(50) \\
12(50)\end{array}$} & \multirow{2}{*}{$\begin{array}{c}\begin{array}{c}\text { Control } \\
\mathbf{N}(\%)\end{array} \\
24(50) \\
\end{array}$} & \\
\hline \multirow{2}{*}{ Gender } & Female & & & \\
\hline & Male & & $12(50)$ & \\
\hline \multirow{2}{*}{ Marital status } & Married & $31(50.8)$ & $30(49.2)$ & \multirow{2}{*}{0.74} \\
\hline & Widow & $5(45.5)$ & $6(54.5)$ & \\
\hline \multirow{2}{*}{ Educational level } & Primary school & $30(51.7)$ & $28(48.3)$ & \multirow{2}{*}{0.55} \\
\hline & High school and more & $6(42.9)$ & $8(57.1)$ & \\
\hline \multirow{2}{*}{ Occupation } & Retired & $12(50)$ & $12(50)$ & \multirow{2}{*}{1} \\
\hline & Housewife & $24(50)$ & $24(50)$ & \\
\hline \multirow{3}{*}{ Financial status } & Bad & $3(37.5)$ & $5(62.5)$ & \multirow{3}{*}{0.01} \\
\hline & Moderate & $32(59.3)$ & $22(40.7)$ & \\
\hline & Good & $1(10)$ & $9(90)$ & \\
\hline \multirow{2}{*}{ Real state ownership } & Sole ownership & $36(51.4)$ & $34(48.6)$ & \multirow{2}{*}{0.49} \\
\hline & Rental & $0(0)$ & $2(100)$ & \\
\hline \multirow{4}{*}{ Living situation } & With spouse & $17(48.6)$ & $18(51.4)$ & \multirow{4}{*}{0.29} \\
\hline & With children & $3(42.9)$ & $4(57.1)$ & \\
\hline & With spouse and children & $12(46.2)$ & $14(53.8)$ & \\
\hline & Alone & $4(100)$ & $0(0)$ & \\
\hline \multirow{2}{*}{ Number of children } & $1-3$ & $13(46.4)$ & $15(53.6)$ & \multirow{2}{*}{0.63} \\
\hline & 4 and more & $23(52.3)$ & $21(47.7)$ & \\
\hline \multirow{2}{*}{$\begin{array}{l}\text { History of chronic } \\
\text { disease }\end{array}$} & No & $31(49.2)$ & $32(50.8)$ & \multirow{2}{*}{1} \\
\hline & Yes & $5(55.6)$ & $4(44.4)$ & \\
\hline
\end{tabular}

The results showed significant differences between the mean insomnia scores in two study groups before the intervention $(\mathrm{p}=0.04)$, but there were more significant differences between the two groups immediately and one month after the intervention $(\mathrm{p}<0.001)$. The mean score of insomnia decreased in intervention group after completion of the reminiscence course while the insomnia score had not noticeable change in control group.

In comparison of insomnia scores between the two study groups before, immediately and one month after the intervention, the intervention group showed significantly less insomnia scores over the time. (Table 3, Figure 2)

Table 3. The mean insomnia scores in 3 time point in intervention and control groups 


\begin{tabular}{|l|c|c|c|c|}
\hline \multirow{2}{*}{ Time } & \multicolumn{3}{|c|}{ Group } & p-value* \\
\cline { 2 - 5 } & Intervention & Control & Total & \\
\hline before the intervention & $16 \pm 1.74$ & $17.38 \pm 3.24$ & $16 \pm 2.67$ & 0.04 \\
\hline immediately after the intervention & $11.27 \pm 2.09$ & $17.38 \pm 3.24$ & $14 \pm 4.1$ & $<0.001$ \\
\hline one month after the intervention & $11.38 \pm 1.93$ & $17.69 \pm 3.01$ & $14 \pm 4.01$ & $<0.001$ \\
\hline
\end{tabular}

*Independent sample t- test

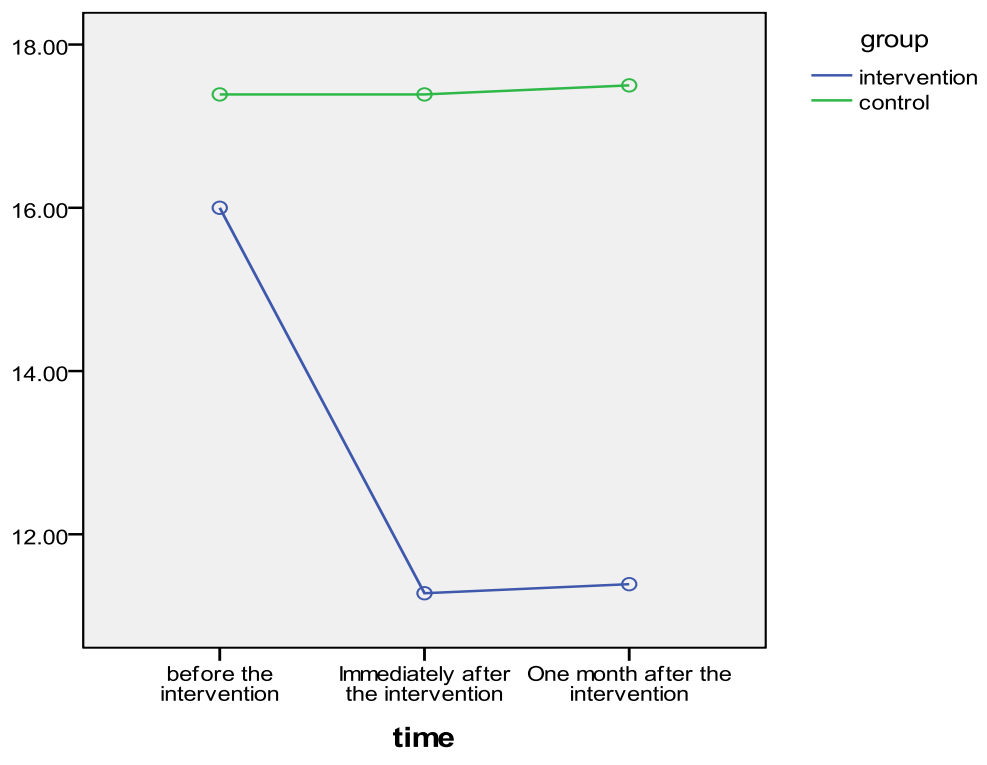

Figure 2. Comparison of mean insomnia scores in 3 time point in two groups

In assessing the effect of reminiscence therapy on insomnia score in the older adults over the time, considering the significant differences in the mean insomnia scores in two study groups at baseline, and also with respect to the significant difference in financial status of two groups, these two variables were considered as confounding variables.

The Mauchly's W test was significant $(p<0.001)$ indicating that the assumption of compound symmetry was not met. Because epsilon was 0.7 , the Greenhouse-Geisser correction results were reported $(F=148.5, p<0.001)$.

The results of repeated measures analysis indicated that the intervention group has significantly less insomnia scores over the time. Although the results of univariate analysis showed a significant difference regarding the financial status between the two groups, however, the insomnia scores differ significantly between two groups; that is, it is not affected by the significant difference in the financial status between the two groups. (Table 4 and 5) 
Table 4. Results of repeated measures analysis related to the effect of time on insomnia scores in two groups

\begin{tabular}{|l|c|c|c|c|}
\hline Source & $\begin{array}{c}\text { Type III Sum of } \\
\text { squares }\end{array}$ & $\begin{array}{c}\text { Mean } \\
\text { Square }\end{array}$ & f & p-value \\
\hline Time & 3.607 & 3.607 & 11.527 & 0.001 \\
\hline $\begin{array}{l}\text { Time* Score of insomnia before the in- } \\
\text { tervention }\end{array}$ & 3.120 & 3.120 & 9.973 & 0.002 \\
\hline Time* Reminiscence & 0.493 & 0.493 & 1.576 & 0.214 \\
\hline Time* Financial status & 0.255 & 0.127 & 0.407 & 0.667 \\
\hline Time* Reminisce* Financial status & 0.038 & 0.019 & 0.061 & 0.941 \\
\hline Error & & & & \\
\hline
\end{tabular}

Table 5. Results of repeated measures analysis related to the effect of the independent variables on insomnia score in two groups

\begin{tabular}{|l|c|c|c|c|}
\hline Source & $\begin{array}{c}\text { Type III Sum } \\
\text { of squares }\end{array}$ & $\begin{array}{c}\text { Mean } \\
\text { Square }\end{array}$ & f & p-value \\
\hline Intercept & 2.047 & 2.047 & 0.459 & 0.501 \\
\hline Score of insomnia before the intervention & 588.462 & 588.462 & 131.930 & 0.000 \\
\hline Reminiscence & 255.844 & 255.844 & 57.359 & 0.000 \\
\hline Financial status & 5.189 & 2.594 & 0.582 & 0.562 \\
\hline Reminisce* Financial status & 0.379 & 0.190 & 0.043 & 0.958 \\
\hline Error & 289.927 & 4.460 & & \\
\hline
\end{tabular}

\section{Discussion}


This study provided useful information about the effect of group reminiscence therapy on insomnia in older adults. Changes in mean score of insomnia had a decreasing trend in the intervention group over the time, while the gradient of insomnia score changes was constant in the control group. This difference is widely attributed to the effective impact of reminiscence therapy on insomnia in this population. During the sessions, participants deliberately recalled the past, listened without any criticism, and expressed their positive feelings about the past, which led to relief of their insomnia. Review of the literature indicated that most of previous studies focused on assessing the impact of group reminiscence therapy on issues such as cognitive function, depression, anxiety and quality of life $(23,25)$ and there were few studies about the effect of reminiscence therapy on sleep phenomenon. One study showed that the quality of sleep increased in the elderly living in nursing homes after a 12-week course of reminiscence therapy (22). The results of another study reported that non pharmacological methods such as reminiscence therapy can significantly reduce the sleep disorders in older adults (41). Group reminiscence therapy has a significant positive impact on mental health and its dimensions such as insomnia and anxiety, social dysfunction and depression in elderly population (30). Also, it is reported the positive impact of reminiscence therapy on depression symptoms such as insomnia and other sleep disorders (42). In assessing the effect of laughter therapy versus group reminiscence therapy on sleep quality of elderly in nursing homes of India, the results indicated that both interventions were significantly effective but laughter therapy had more impact on sleep quality in elderly (33).Chang and Chien (2018) assessed the impact of group reminiscence intervention on depression in older adults with dementia using the Cornell Scale for Depression in Dementia (CSDD). They evaluated sleep disorders as one of the subsets of this scale and they did not find any highlighted decrease in sleep disorders of these people (32). Furthermore, in assessment of sleep disorders in older patients with Alzheimer's disease, researchers did not find a significant change due to reminiscence intervention (31).

A large number of older adults suffer from insomnia or they may have problems in sleep onset and sleep maintenance. These disorders may increase their daytime fatigue so they can't do their activities of daily living. Group reminiscence therapy seems to alleviate these problems to some extent, before they need to take medication. Discussing about various topics in the same group of people with similar concerns, can help to deal with past and future problems and live in present moment. In order to clarify the mechanism of the influence of group reminiscence intervention, it 
can be notified that older adults express their talents and values and try to have more emphasis on their precious acts and their problem-solving approaches during their life (29).In reminiscence intervention, there is always focus on the positive interactions and it allows people to talk about what is important to them. It provides a comfortable and supporting environment and improves the communication, sense of belonging and self-confidence. It seems that the older adults express their positive feelings by reminiscing the past and this dramatically leads to emotional release and improves sleep quality. However, the type of measurement tool for assessing the reminiscence therapy effects and the related factors, the type of reminiscence intervention, the protocol and way of organizing the sessions and the time, location and consistency of the intervention are all effective to the results of the intervention (29).

This study has some limitations. First, the participants were all selected from a single health care center. Second, it had a short follow-up period following the reminiscence therapy. Also, participants in this study were young older adults that may be in comparing to old older adults, have lower level of insomnia. This can lead to low generalizability of the results of this study.

\section{Conclusion}

Findings of this study showed that reminiscence therapy intervention decreases the severity of insomnia in older adults. Therefore, it can be applied as an easy, applicable and low-cost technique in health care facilities and it is expected to be welcomed by older adults, their families and health care providers.

Considering that in this study, the severity of insomnia was evaluated one month after termination of the intervention, it is recommended to conduct studies with longer follow up periods (more than one month) to compare with the results of the present study.

\section{Acknowledgements}

We as authors of this paper would like to thanks all the older adults participating in this research. The authors also appreciate from Kashan University of Medical Sciences for financial support of this study.

\section{References}


1. Rawlins RP, Williams SR, Beck CK. Mental health-psychiatric nursing: a holistic life-cycle approach. St. Louis, MO: Mosby; 1993.

2. Åkerstedt T, Axelsson J, Lekander M, Orsini N, Kecklund G. Do sleep, stress, and illness explain daily variations in fatigue? A prospective study. J Psychosom Res. 2014; 76(4):280-85. [DOI:10.1016/j.jpsychores.2014.01.005]

3. Veauthier C, Paul F. Sleep disorders in multiple sclerosis and their relationship to fatigue. Sleep Med. 2014; 15:5-14. [DOI:10.1016/j.sleep.2013.08.791]

4. Almklov EL, Drummond SPA, Orff H, Alhassoon OM. The effects of sleep deprivation on brain functioning in older adults. Behav Sleep Med. 2015; 13(4):324-45. [DOI: 10.1080/15402002.2014.905474]

5. Patel D, Steinberg J, Patel P. Insomnia in the elderly: a review. J Clin Sleep Med. 2018; 14(6):1017-24. [DOI:10.5664/jcsm.7172]

6. Cooke JR, Ancoli-Israel S. Normal and abnormal sleep in the elderly. Handbook of Clinical Neurology. 2011; (98): 653-65. [DOI:10.1016/B978-0-444-52006-7.00041-1]

7. American Psychiatric Association. Diagnostic and Statistical Manual of Mental Disorders. 5th ed. Arlington, VA: American Psychiatric Publishing; 2013. [DOI:10.1176/appi.books.9780890425596]

8. Crowely K. Sleep and sleep disorders in older adults. Neuropsychology Review. 2011; 21: 4153. [DOI: 10.1007/s11065-010-9154-6]

9. Buysse DJ, Germain A, Moul DE, Franzen PL, Brar LK, Fletcher ME, et al. Efficacy of brief behavioral treatment for chronic insomnia in older adults. Arch Intern Med. 2011; 171(10):88795. [DOI:10.1001/archinternmed.2010.535]

10. Morin CM, Colecchi C, Stone J, Sood R, Brink D. Behavioral and pharmacological therapies for late-life insomnia: a randomized controlled trial. JAMA. 1999; 281:991-99. [DOI:10.1001/jama.281.11.991]

11. Li J, Yao YS, Dong Q, Dong YH, Liu JJ, Yang LS, et al. Characterization and factors associated with sleep quality among rural elderly in China. Arch Gerontol Geriatr. 2013; 56:23743. [DOI:10.1016/j.archger.2012.08.002]

12. Kunz D, Mahlberg R. A two- part, double- blind, placebo- controlled trial of exogenous melatonin in REM sleep behavior disorder. J Sleep Res. 2010; 19(4):591-96. [DOI:10.1111/j.1365-2869.2010.00848.x]

13. Beck JS. Cognitive-behavioral therapy. Clinical textbook of addictive disorders; 2011. P. 474-501. 
14. Wang JJ. Group reminiscence therapy for cognitive and affective function of demented elderly in Taiwan. Int J Geriatr Psychiatry. 2007; 22:1235-40. [DOI:10.1002/gps.1821]

15. Woods B, O'Philbin L, Farrell EM, Spector AE, Orrell M. Reminiscence therapy for dementia.Cochrane database syst rev. 2018;3. [DOI:10.1002/14651858.CD001120.pub3]

16. Chen TJ, Li HJ, Li J. The effects of reminiscence therapy on depressive symptoms of Chinese elderly: study protocol of a randomized controlled trial. BMC Psychiatry. 2012; 12:189. [DOI: 10.1186/1471-244X-12-189]

17. Ando M, Morita T. Efficacy of the structured life review and the short-term life review on the spiritual well-being of terminally ill cancer patients. Health. 2010; 2(4):342-46. [DOI:10.4236/health.2010.24051]

18. Sheibani Tezerji F, Pakdaman SH, Hassanzadeh Tavakoli MR. The effect of reminiscence on depression and loneliness in elderly. J Appl Psychol.2010; 4(13):26-39.

19. Wu CH, Yao G. Psychometric analysis of the short-form UCLA loneliness scale (ULS-8) in Taiwanese undergraduate students. Pers Individ Dif. 2008; 44(8):1762-71. [DOI:10.1016/j.paid.2008.02.003]

20. Pinquart M, Forstmeier S. Effects of reminiscence interventions on psychosocial outcomes: a meta-analysis. Aging \&Ment Health. 2012; 16(5):541-58. [DOI: 10.1080/13607863.2011.651434]

21. Cappeliez P, Rivard V, Guindon S. Functions of reminiscence in later life: proposition of a model and applications. European review of applied psychology. 2007; 57(3):151-56. [DOI:10.1016/j.erap.2005.02.014]

22. Aşiret GD. Effect of reminiscence therapy on the sleep quality of the elderly living in nursing homes: A randomized clinical trial. Eur J Integr Med. 2018; 20:1-5. [DOI:10.1016/j.eujim.2018.03.007]

23. Sotodeh Navrodi SO, Pooragha Roodbordeh F, Kafi SM, Poornesaii, GS. Effectiveness of reminiscence group therapy on male elderly's mental health. J Guilan Uni Med Sci. 2013; 22(85):61-7.[Persian]

24. Gaggioli A, Scaratti C, Morganti L, Stramba-Badiale M, Agostoni M, Spatola CA, et al. Effectiveness of group reminiscence for improving wellbeing of institutionalized elderly adults: study protocol for a randomized controlled trial. Trials. 2014; 15:408. [DOI: 10.1186/1745-6215$\underline{15-408]}$

25. Zhang SJ, Hwu YJ, Wu PI, Chang CW. The Effects of reminiscence therapy on depression, self-esteem and life satisfaction on institutionalized older adults: a meta-analysis. J Nurs Healthc Res. 2015; 11:33-42. 
26. Ghanbarpanah I, Fallahi Khoshknab M, Maddah S, Mohammadi F, Khodaee M. The Effects of the group reminiscence on cognitive status of mild cognitive impaired elders. IJPN. 2014; 1(4):1-12. [Persian]

27. Chiang KJ, Chu H, Chang HJ, Chung MH, Chen $\mathrm{CH}$, Chiou HY, et al. The effects of reminiscence therapy on psychological well- being, depression, and loneliness among the institutionalized aged. Int J Geriatr Psychiatry. 2010; 25(4):380-8. [DOI:10.1002/gps.2350]

28. Hojjati H, Akhonzadeh G, Hassanalipour S, Sharifnia SA, Asayesh H. The effect of reminiscence on the amount of group self-esteem and life satisfaction of the elderly. The J Urmia Nurs Midwifery Fac. 2011; 9(5):350-6. [Persian]

29. Yousefi Z, Sharifi K, Tagharrobi Z, Akbari H.The effect of narrative reminiscence on happiness of elderly women. Iran Red Crescent Med J. 2015; 17(11): e19612. [DOI:10.5812/ircmj.19612]

30. Musavi M, Mohammadian S, Mohammadinezhad B. The effect of group integrative reminiscence therapy on mental health among older women living in Iranian nursing homes. Nurs Open. 2017; 4(4):303-9. [DOI:10.1002/nop2.101]

31. Duru Aşiret G, Kapucu S. The effect of reminiscence therapy on cognition, depression, and activities of daily living for patients with Alzheimer disease. J Geriatr Psychiatry and Neurology. 2016; 29:31-7. [DOI: 10.1177/0891988715598233]

32. Chang H, Chien HW. Effectiveness of group reminiscence therapy for people living with dementia in a day care centers in Taiwan. Dementia. 2018; 17(7): 924-35. [DOI: $10.1177 / 1471301217725185]$

33. Jebapriya CH, Aras R, Devadason JM. Eff ectiveness of laughter therapy versus reminiscence therapy on quality of sleep, among senior citizens living in old age homes in Coimbatore, Tamilnadu. Int J Sci Res. 2015; 4:3-5.

34. Musavi M, Sheikhi N, Sheikhi S, Hemmati Maslakpak M. The effect of reminiscence group therapy on the depression of the elderlies in nursing homes of Urmia. J Urmia Nurs Midwifery Fac. 2013; 11(7): 507-15.

35. Folstein MF, Folstein SE, McHugh, PR. "Mini-mental state". A practical method for grading the cognitive state of patients for the clinician. J Psychiatr Res. 1975; 12(3):189-98. [DOI: $\underline{10.1016 / 0022-3956(75) 90026-6]}$

36. Shafaat N, Makvand Hosseini S, Rezaei AM. Effectiveness of Multi-components cognitivebehavioral therapy on insomnia intensity and quality of sleep among breast cancer patients after surgery. J Res Psychol Health. 2017; 11(2):82-91. [DOI:10.29252/rph.11.2.82]

37. Seyedian M, Falah M, Nourouzian M, Nejat S, Delavar A, Ghasemzadeh HA. Validity of the Farsi version of mini-mental state examination. Sci J Iran Med Council. 2008; 25(4):408-14. 
38. Bastien $\mathrm{CH}$, Vallières A, Morin CM. Validation of the Insomnia Severity Index as an outcome measure for insomnia research. Sleep Med. 2001; 2(4):297-07. [DOI: 10.1016/S13899457(00)00065-4]

39. Yazdi Z, Sadeghniiat-Haghighi K, Zohal MA, Elmizadeh K. Validity and reliability of the Iranian version of the Insomnia Severity Index. MJMS. 2012; 19(4):31-6.

40. Stinson CK, Kirk E. Structured reminiscence: an intervention to decrease depression and increase self- transcendence in older women. J ClinNurs. 2006; 15(2): 208-18. [DOI:10.1111/j.1365-2702.2006.01292.x]

41. Rawtaer I, Mahendran R, Chan HY, Lei F, Kua EH. A nonpharmacological approach to improve sleep quality in older adults. AsiaPac Psychiatry. 2018; 10:e12301. [DOI:10.1111/appy.12301]

42. Wang JJ, Hsu YC, Cheng SF. The effects of reminiscence in promoting mental health of Taiwanese elderly. Int J Nurs Stud. 2005; 42:31-6. [DOI:10.1016/j.ijnurstu.2004.05.010]

\section{Bibliographic information of this paper for citing:}

Sahragard F, Rezaei M, Izadi-Avanji F.S, et al. The effect of group reminiscence therapy on insomnia in older adults: A randomized controlled clinical trial

J Res Dev Nurs Midw, 2020; 17(1): 94-110.

Copyright $\odot$ 2020, Fatemeh Sahragard, Mahboubeh Rezaei, Fatemeh Sadat Izadi-Avanji, Fatemeh Atoof, Maryam Ahmadishad. 